\title{
Quasi-optical approach to reconstruction of plasma fluctuations using amplitude distribution of transmitted microwave beam
}

\author{
E.D. Gospodchikov, D.I. Sobolev, T.A. Khusainov, A.A. Balakin, A.G. Shalashov \\ Institute of Applied Physics of RAS, Nizhny Novgorod, Russia, egos@appl.sci-nnov.ru
}

\section{Introduction}

Despite considerable progress in the theoretical understanding of confined plasmas, there is still a need for a detailed description of the fundamental physical parameters playing a role in the processes governing equilibrium, stability, and transport. Turbulence properties are some of the most important of such parameters.

Two basic approaches are used for remote sensing of the density fluctuation by electromagnetic beams. The first one is based on the microwave reflectometry which basically allows the reconstruction of the turbulence spectrum by the spectrum of the scattered signal in the far zone [1]. The second one is phase contrast imaging (PCI), basing on reconstruction of the turbulence distribution by phase distortion of passing laser beams in geometrical optics zone [2]. In this work we present a theoretical approach to the reconstruction of inhomogeneous plasma properties by amplitude distribution of microwave beam measured after pass through the plasma in intermediate i.e. Fresnel zone. First examples of reconstructions by this method are also presented. There are two promising advantages of this approach. First, the measurement of amplitude distribution in the beam transmitted through the plasma can be done easier than phase measurements required for PCI, because it does not require a matrix of separate receivers for each transverse point hence potentially has better spatial resolution. Second, amplitude perturbations in the Fresnel zone are of the first order of phase velocity perturbations, and the technique might allow detection fluctuations with low amplitude leading to weak and/or low angle scattering.

The discussed method is based on the quasi-optical approach to modeling the propagation of wave beams developed at IAP RAS, which allows taking into account the simultaneous effects of inhomogeneity and spatial dispersion of the medium, diffraction, aberration and dissipation of the wave beam. Based on this approach, a numerical code LAQO was developed. The code is able to model the propagation and the electron cyclotron absorption of wave beams in weakly relativistic plasma in electromagnetically large devices (with typical size of few thousands of wavelengths) in a reasonable time. Calculations using LAQO proved the importance of taking into account these effects to describe correctly the RF power deposition profile in tokamak plasmas [3, 4] and in open magnetic trap $[5,6]$. A small solution time for the direct problem of wave beam propagation allows the use of LAQO to solve the inverse problem, such as reconstruction of distributions of fluctuating plasma parameters using the amplitude profile of the transmitted beam. To solve the inverse problem we adopt the iterative method for the synthesis of the surface of the passive elements of microwave transmission systems developed in IAP RAS, which allows one to achieve extremely high computational precision using a several iterations of direct problem calculations [7]. Note that this technique requires knowledge of phase distribution for the incident wave beam only, and for the transmitted wave beam only intensity distribution is needed.

\section{Reconstruction algorithm}

As the first step we present the results of reconstruction of the parameters of single perturbation of refraction coefficient in homogeneous isotropic media $\delta n=\delta_{0} \exp \left(-\left(\vec{r}-\vec{r}_{0}\right)^{2} / a^{2}\right)$. This simplest plasma density perturbation is characterized by amplitude $\delta_{0}$, position $\vec{r}_{0}$ and width $a$.

The reconstruction routine uses the following algorithm. Given the amplitude and phase of the wave beam $\left(A_{\text {in }}, \phi_{\text {in }}\right)$ specified in start plane, a "measureable" amplitude distribution $A_{\text {out }}$ in measurement plane is calculated for a wave beam transmitted through the media with refraction coefficient perturbation. Then the same calculation is done for the unperturbed media, and output amplitude and phase distributions $\left(A_{\text {out }}^{i}, \phi_{\text {out }}^{i}\right)$ are obtained. After that, a substitution $A_{\text {out }}^{i} \rightarrow A_{\text {out }}$ is done, and the beam propagation is calculated in reverse direction. The amplitude distribution obtained in the latter calculation $A_{i n}^{i}$ is substituted with the one taken from the incident beam, and the difference of phase distributions $\phi_{i n}^{i}-\phi_{i n}$ is stored as the phase corrector. This sequence is repeated until the amplitude distribution calculated with phase corrector and "measured" amplitude distribution converges with enough precision. Resulting phase corrector is converted into refraction coefficient perturbation. Note that distributions along and transversely to the beam are reconstructed essentially differently. Due to the fact that the phase corrector is 2-dimensional phase perturbation, the distribution along the beam is reconstructed ambiguously. Therefore we have to use an additional scan over the position of the phase corrector for reconstruction of the longitudinal distribution of fluctuation.

\section{Transverse distribution of refraction coefficient}

Reconstructed refraction coefficient with parameters $\delta_{0}=0.05, a=1 \mathrm{~cm}$ is presented in figure 1 . The wavelength was taken $\lambda=0.5 \mathrm{~cm}$, quasioptical beam size at input $a_{\text {beam }}=3 \mathrm{~cm}$, the length of propagation track is $l=30 \mathrm{~cm}$. Refraction coefficient perturbation is located on the beam axis. 

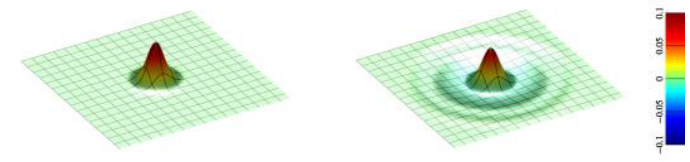

Fig. 1. Reconstruction of undisplaced perturbation. Right panel - distribution of refraction coefficient perturbation; left panel reconstructed distribution of refraction coefficient perturbation
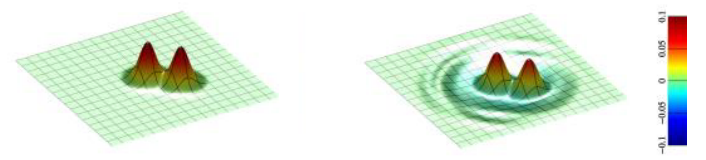

Fig. 2. Reconstruction of refraction coefficient perturbation with two maximums. Right panel - distribution of refraction coefficient perturbation; left panel - reconstructed distribution of refraction coefficient perturbation

One can see that position, amplitude and shape of perturbation in transverse plane are reconstructed with high precision, and resolution is less than the beam aperture. To illustrate this point, the reconstruction of "twohump" perturbation is presented in figure 2 .

As one can see, it is possible to reconstruct the fluctuation parameters with precision comparable to the wavelength.

\section{Longitudinal position of perturbation}

Proposed reconstruction procedure result in 2-dimensional phase corrector, which can be converted into refraction coefficient perturbation distribution with longitudinal uncertainty. It is unavoidable limitation because the original inverse problem is formally incorrect. Our calculations, however, give some hope that the longitudinal position can be reconstructed with precision about the wave beam waist. To illustrate this, we made calculations with the same parameters as presented in figure 1 , but with a shifted position of the phase corrector along the beam path back and forth from the position of the perturbation. The results of modeling for phase corrector shifted relatively right position are presented in figure 3. Comparing figures 1 and 3 one can see that "inaccuracy" of the phase corrector position leads to the emergence of a strong "Fresnel lens" in the reconstructed phase corrector. Therefore, the condition of minimization of this "lens" allows reconstruction of longitudinal position of perturbation.

This method does not give the accuracy of the reconstruction of the longitudinal coordinate comparable to the accuracy of the reconstruction of the transverse distribution, but it allows to improve accuracy of reconstruction.
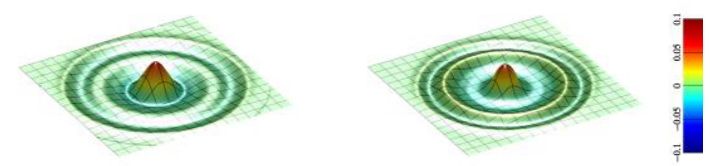

Fig. 3. Reconstruction results with different positions of phase corrector along the beam path. Right panel - before right position; left panel - after right position
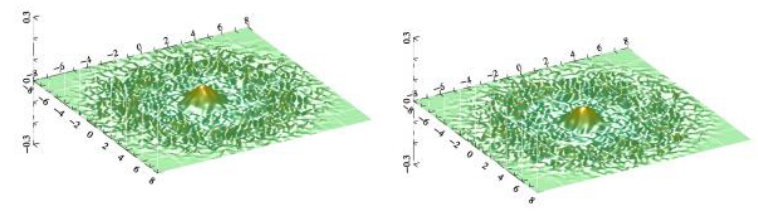

Fig. 4. Reconstruction of perturbation using noised transmitted beam distribution. Right panel after 10 procedure iterations.; left panel -40 iteration

\section{Transmitted beam with noised intensity distribution}

Intensity distribution can be measured with limited accuracy. We demonstrated possibility of fluctuation reconstruction using noised (by $10 \%$ incoherent noise) "measured" distribution (figure 4).

This work was supported by RFBR grant № 15-0207600 .

\section{References}

1. Skvortsova N.N., Akulina D.K., Batanov G.M., Kharchev N.K., Kolik L.V., Kovrizhnykh L.M., Letunov A.A., Logvinenko V.P., Malakhov D.V., Petrov A.E. Effect of ECRH regime on characteristics of short-wave turbulence in plasma of the L-2M stellarator // Plasma Phys. Control. Fusion. 2010. V. 52. P. 055008.

2. Marinoni A., Coda S., Chavan R., and Pochon G. Design of a tangential phase contrast imaging diagnostic for the TCV tokamak // Rev. of Scientific Instr. 2006. V.77. P. 10E929.

3. Bertelli N., Balakin A.A., Westerhof E. and Buyanova M.N. ECCD calculations in ITER by means of the quasioptical code// Nucl. Fusion, 2010, V. 50, P. 115008.

4. Maj O., Balakin A.A., Poli E. Effects of aberration on paraxial wave beams: beam tracing versus quasi-optical solutions // Plasma Phys. Controll. Fusion. 2010. V. 52, P. 085006.

5. Shalashov A. G., Balakin A. A., Gospodchikov E. D., and Khusainov T.A. Quasi-optical theory of microwave plasma heating in open magnetic trap// Physics of Plasmas 2016, V. 23, P. 112504.

6. Shalashov A.G. Balakin A.A. Khusainov T.A. Gospodchikov E. D. Solomakhin A.L. Quasi-optical simulation of the electron cyclotron plasma heating in a mirror magnetic trap // JETP, 2017. Vol. 124, No 2, P 325.

7. Sobolev D.I., Denisov G.G. Principles of Synthesis of Multimode Waveguide Units // IEEE Transactions on Plasma Science, 2010, V.38, No.10, P. 2825. 